\title{
Quo vadis Dermatohistology?
}

Wie wichtig die Dermatohistologie in der täglichen Praxis ist, braucht man einem Dermatologen nicht zu erklären. Auch wenn eine Biopsie nicht jede Frage beantworten kann - der dermatohistologische Befund ist häufig das Teil in unserem diagnostischen Puzzle, auf das es ganz besonders ankommt. Von der klinisch-histologischen Korrelation, der Zusammenschau von makroskopischem und mikroskopischem Befund, profitieren dabei beide, Kliniker und Histologe, und häufig sind die Funktionen ja auch in Personalunion vereint.

Die Bedeutung dieser Zusammenschau haben schon unsere dermatologischen Vorväter im 19. Jahrhundert erkannt. Deshalb entstand die Dermatohistologie in und aus der klinischen Dermatologie. Dass Dermatohistologie eine primär dermatologische Disziplin ist, zeigt sich auch in den Lehrbüchern - sie wurden ausschließlich von Dermatologen geschrieben; von pathologischer Seite findet sich wenig. Bedauerlicherweise wurde in den letzten Jahren die Dermatohistologie in manchen Hautkliniken nicht mit der Liebe gepflegt, die sie verdient, dies vielleicht unter anderem deshalb, weil sie unter Forschungsgesichtspunkten keine hohen Impact-Faktoren verspricht, sondern eine große Erfahrung voraussetzende klinische Routine-Leistung darstellt.

Wie es in Zeiten von Verteilungskämpfen nicht anders sein konnte, wurde die Dermatohistologie gleichzeitig Streitobjekt zwischen ärztlichen Disziplinen, wobei Vertreter der Pathologie Dermatologen die Kompetenz auf diesem Gebiet absprachen. Umso erfreulicher war es, dass sich die Berufsverbände und wissenschaftlichen Fachgesellschaften der Dermatologen und der Pathologen schon vor über zwei Jahren auf die Kriterien für eine fakultative Weiterbildung in der Histologie der Haut geeinigt haben, zunächst bezogen auf die derzeit gültige Weiterbildungsordnung, aber auch ausgerichtet auf die derzeit verhandelte Neufassung der Weiterbildungsbestimmungen.

Der Inhalt dieses wichtigen Papiers ist bisher leider nur wenigen Dermatologen bekannt. Danach muss ein Dermatohistologe eine mindestens zweijährige ganztägige Weiterbildung an einer berechtigten Weiterbildungsstätte abgeleistet haben, wovon ein halbes Jahr auf die Gebietsweiterbildung zum Hautarzt angerechnet werden kann, und mindestens 6000 dermatohistologische Präparate selbstständig untersucht und befundet haben. Hierzu gehören konventionelle und spezielle Methoden wie die Spezialfärbungen, die Immunhistologie etc.

Für die Weiterbildungsstätte gelten spezifische Kriterien: Die Einrichtung muss von einem Arzt geleitet werden, der die Berechtigung zur Durchführung der genannten Untersuchungen erworben hat und danach mindestens zwei Jahre in der Diagnostik der Histologie der Haut tätig gewesen ist. Es müssen jährlich mindestens 6000 dermatohistologische Krankheitsfälle diagnostiziert werden, eine Lehrsammlung muss vorhanden sein, die Einrichtung muss an dermatohistologisch-klinischen Demonstrationen teilnehmen oder diese durchführen und sich an qualitätssichernden Massnahmen beteiligen.

Auch wenn die Inhalte dieses Papiers bisher noch nicht in die Weiterbildungsordnung übersetzt wurden - sie können als vereinbarte Kriterien gelten, wem man dermatohistologische Kompetenz zutrauen kann. Es ist nun an uns Dermatologen, diese Kriterien umzusetzen und die Dermatohistologie wieder als einen Schwerpunkt unseres Faches zu pflegen. Sie sollte wichtige Säule der dermatologischen Weiterbildung selbst für die Kollegen sein, die nicht Dermatohistologen werden wollen. Von der klinischhistologischen Korrelation profitiert eben nicht nur der befundende Histologe, sondern auch der dermatologische Kliniker.

Wenn die Dermatohistologie ein integraler Teil der Dermatologie bleiben soll, dann muss sie auch in Kliniken und Praxen kompetent gelehrt werden. Da nicht alle Kliniken aus eigenem Material die genannten 6000 Präparate jährlich aufbringen können, sind sie auf Einsendungen angewiesen. Hier ist die Solidarität der niedergelassenen Dermatologen gefordert, um unserem Nachwuchs den Einstieg in dieses wichtige und traditionsreiche Gebiet unseres Faches zu ermöglichen. 